\title{
The diagnostic significance of 64-slice spiral CT combined with serological CA19-9, Bcl-2, CYFRA21-1 detection in thoracic esophageal carcinoma
}

\author{
Junfei Hao ${ }^{1 \#}$, Wei Liu ${ }^{2 *}$, Chunyan Zhao ${ }^{2}$, Taiyu $\mathrm{Xia}^{2}$ \\ ${ }^{1}$ Department of Medical Equipment, Dazu District People's Hospital, Chongqing, China; ${ }^{2}$ Department of Oncology, Dazu District People's Hospital, \\ Chongqing, China \\ Contributions: (I) Conception and design: J Hao, W Liu; (II) Administrative support: T Xia; (III) Provision of study materials or patients: C Zhao; (IV) \\ Collection and assembly of data: J Hao, W Liu; (V) Data analysis and interpretation: J Hao, W Liu; (VI) Manuscript writing: All authors; (VII) Final \\ approval of manuscript: All authors. \\ "These authors contributed equally to this work. \\ Correspondence to: Chunyan Zhao; Taiyu Xia. Department of Oncology, Dazu District People's Hospital, No. 1073 South Second Ring Road, \\ Tangxiang Street, Dazu District, Chongqing 402360, China. Email: zcy513568606@163.com; xiataiyu500228@163.com.
}

Background: To analyze the clinical value of multi-slice spiral computed tomography (MSCT) combined with carbohydrate antigen 19-9 (CA19-9), B-cell leukemia/lymphoma-2 protein (Bcl-2), and cytokeratin 19 fragment antigen 21-1 (CYFRA21-1) detection in the diagnosis of thoracic esophageal cancer.

Methods: The clinical data of 74 patients with thoracic esophageal cancer admitted to the Dazu District People's Hospital in Chonqing, China, from December 2019 to December 2020 were collected (esophageal cancer group), and their computed tomography (CT) signs were analyzed. Another 55 healthy people who underwent physical examination during the same period in the hospital were selected for the healthy group. The serum levels of CA19-9, Bcl-2, and CYFRA21-1 in the different populations were compared, using the receiver operating characteristic (ROC) curve to analyze the value of MSCT combined with CA19-9, Bcl-2, and CYFRA21-1 detection in the diagnosis of thoracic esophageal cancer.

Results: The serum levels of CA19-9, Bcl-2, and CYFRA21-1 in patients of the esophageal cancer group were significantly higher than those in the healthy group $(\mathrm{P}<0.05)$. The serum levels of CA19-9, Bcl-2, and CYFRA21-1 in patients with poorly differentiated, stage III-IV carcinoma and lymph node metastasis were significantly higher than in those patients with moderately well-differentiated, stage I-II carcinoma and no lymph node metastasis $(\mathrm{P}<0.05)$. The CT scans of patients in the esophageal cancer group showed esophageal walls with irregular, needle-shaped, circular, or localized eccentric thickening and narrowed lumens, which were dilated above the cancerous lesions. Some tumors compressed adjacent organs to deform and shift the organs, resulting in the disappearance of surrounding fat layers. Enhanced scans showed mild or moderate enhancement, with large-diameter lesions unable to enhance central, low-density, necrotic areas. The ROC curve showed that the area under the curve (AUC) and the sensitivity and specificity of MSCT combined with CA19-9, Bcl-2, and CYFRA21-1 detection were all higher than for esophageal lesions detected by individual indicators.

Conclusions: CA19-9, Bcl-2, and CYFRA21-1, which are abnormally expressed in patients with esophageal cancer, may be related to the occurrence and development of esophageal cancer. MSCT combined with CA199, Bcl-2, and CYFRA21-1 detection appears to enhance the diagnosis of esophageal cancer.

Keywords: 64-slice spiral CT; carbohydrate antigen 19-9 (CA19-9); B-cell leukemia/lymphoma-2 (Bcl-2) gene; cytokeratin fragment 21-1; thoracic esophageal carcinoma

Submitted Oct 31, 2021. Accepted for publication Dec 06, 2021.

doi: $10.21037 /$ tcr-21-2522

View this article at: https://dx.doi.org/10.21037/tcr-21-2522 


\section{Introduction}

Esophageal cancer is a common malignant tumor of the digestive tract, with high mortality and morbidity. According to clinical data, esophageal cancer is more common in middle-aged and elderly people (1). However, due to the nonspecific symptoms of esophageal cancer in the early stages of disease and the rapid progression of the disease, it is difficult to diagnose early in the clinic. Previous studies have found that the 5 -year survival rate of patients with advanced esophageal cancer after surgical treatment is approximately $30 \%$. If early surgical treatment is performed on esophageal cancer patients, the 5 -year survival rate can be as high as $70 \%(2,3)$. Therefore, identifying techniques to enhance the early diagnosis of esophageal cancer is a key focus of current clinical research.

Computed tomography (CT) has been widely used in the clinical diagnosis of esophageal cancer due to its high-density resolution and multi-directional continuous scanning, however the false positive rate and false-negative rate of esophageal cancer diagnosis in daily practice impacts the quality of CT diagnosis (4). Tumor markers are also a hot topic in the early diagnosis of esophageal cancer, having high sensitivity and specificity, however there are certain limitations in defining lesion location (5). There are many reports on the diagnosis of esophageal cancer by CT or tumor markers, but there are few reports on the diagnosis of esophageal cancer by ROC curve analysis imaging combined with tumor markers. In this study, the clinical value of multi-slice spiral CT (MSCT) combined with the detection of the tumor markers, carbohydrate antigen 19-9 (CA19-9), B cell leukemia/lymphoma-2 gene (Bcl-2), and cytokeratin 19 fragment antigen 21-1 (CYFRA21-1) in the diagnosis of thoracic esophageal cancer were studied to provide a scientific basis for clinical diagnosis and treatment. We present the following article in accordance with the STARD reporting checklist (available at https:// dx.doi.org/10.21037/tcr-21-2522).

\section{Methods}

\section{General information}

The clinical data of 74 patients with thoracic esophageal cancer admitted to the Dazu District People's Hospital in Chonqing, China, from December 2019 to December 2020 were collected. Inclusion criteria were that patients had: (I) accurate pathological results as obtained by biopsy or surgery, (II) complete clinical data, (III) no other co- existing benign or malignant diseases, and (IV) never received radiotherapy or chemotherapy. Exclusion criteria for patients were: (I) recent use of hormone drugs and/ or immune system regulators, (II) CT examination contraindications, (III) severe cognitive and communication impairment, and (IV) secondary recurrence.

A total of 74 patients with esophageal cancer were enrolled into the study, including 53 males and 21 females aged from 31 to 78 years with an average age of $54.23 \pm 15.16$ years. The patients were distributed across the clinical stages as follows: stage I (6 cases), stage II (16 cases), stage III ( 25 cases), and stage IV (27 cases). The patients had the following pathological types of cancer: squamous cell carcinoma (23 cases) and non-squamous cell carcinoma (51 cases). There were 53 cases of lymph node metastasis, distributed as poorly differentiated (41 cases) and moderately and highly differentiated (33 cases). The healthy group of 55 healthy people who underwent physical examination in the hospital during the same period consisted of 40 males and 15 females, aged 31 to 79 years, with an average age of $53.45 \pm 14.12$ years. There was no significant difference between the two groups $(\mathrm{P}>0.05)$. All procedures performed in this study involving human participants were in accordance with the Declaration of Helsinki (as revised in 2013). The study was approved by ethics committee of Dazu District People's Hospital (No. EC2020-031-05) and informed consent was taken from all the patients.

\section{Study methods}

\section{MSCT examination}

The examination instrument used was the Discovery CT750 HD (GE Healthcare), with the patient in the supine position for the line scan and enhanced scan. Scanning parameters were: tube voltage $120 \mathrm{kV}$, tube current $280 \mathrm{~mA}$, scanning layer thickness and spacing $5 \mathrm{~mm}$, field of vision $28 \mathrm{~cm} \times 35 \mathrm{~cm}$, and matrix $512 \times 512$. A conventional plain scan was performed followed by an enhanced scan $(80 \mathrm{~mL}$ iohexol, injection rate of $2.5 \mathrm{~mL} / \mathrm{s}$ ), with a delayed scan time of $30 \mathrm{~s}$. A $3 \mathrm{D}$ reconstruction of the image occurred after scanning.

\section{Tumor marker examination}

Examination of the tumor markers was composed of two parts: (I) sample collection and processing, in which $5 \mathrm{~mL}$ of venous blood were extracted from the patients, on an empty stomach, and centrifuged at $4{ }^{\circ} \mathrm{C}$. After 3,000 r/min 
Table 1 Comparison of CA19-9, Bcl-2, and CYFRA21-1 levels between the two group

\begin{tabular}{lcccc}
\hline & Esophageal cancer group $(\mathrm{n}=74)$ & Healthy group $(\mathrm{n}=55)$ & $\mathrm{t}$ & $\mathrm{P}$ \\
\hline CA19-9 $(\mathrm{U} / \mathrm{mL})$ & $33.04 \pm 10.32$ & $18.57 \pm 7.07$ & 9.443 & $<0.001$ \\
BCl-2 $(\mathrm{ng} / \mathrm{mL})$ & $17.67 \pm 4.87$ & $11.19 \pm 6.52$ & 6.186 & $<0.001$ \\
CYFRA21-1 $(\mu / \mathrm{mL})$ & $5.14 \pm 2.95$ & $2.34 \pm 0.6$ & 7.953 & $<0.001$ \\
\hline
\end{tabular}

CA19-9, carbohydrate antigen 19-9; Bcl-2, B-cell leukemia/lymphoma-2 protein; CYFRA21-1, cytokeratin 19 fragment antigen 21-1.

for $10 \mathrm{~min}$, the upper serum was separated and immediately sent for examination or stored at $-20^{\circ} \mathrm{C}$ for examination; (II) detection methods, in which CA19-9 and CY-FRA21-1 were detected by electrochemiluminescence, using kits provided by Shanghai Roche Diagnostic Products Co., Ltd. (China). Bcl-2 was detected by an enzyme-linked immunosorbent assay, using the kit provided by Abbott Laboratories Trading Co., Ltd. (Shanghai, China).

\section{Observational indicators}

In this study: (I) the serum levels of CA19-9, Bcl-2, and CYFRA21-1 were compared between the two groups; (II) the serum levels of CA19-9, Bcl-2, and CYFRA21-1 in esophageal cancer patients with different clinical features were compared; (III) the size, shape, density, and other CT signs of esophageal carcinoma were observed; and (IV) the diagnostic value of MSCT combined with CA19-9, Bcl-2, and CYFRA21-1 detection in esophageal cancer was assessed.

\section{Statistical method}

The data were analyzed using SPSS v 20.0 statistical software. Measurement data were described using mean and standard deviation $(\bar{x} \pm S)$, and the $t$-test was used for a comparison between the groups. The enumeration data were expressed as $\mathrm{n}(\%)$, and the chi-squared $\left(\chi^{2}\right)$ test was performed. The receiver operating characteristic (ROC) curve was used to analyze the value of MSCT combined with CA19-9, Bcl-2, and CYFRA21-1 detection in the diagnosis of esophageal cancer. $\mathrm{P}<0.05$ was considered statistically significant.

\section{Results}

Comparison of CA19-9, Bcl-2, and CYFRA21-1 levels between the two groups

The serum levels of CA19-9, Bcl-2, and CYFRA21-1 in patients of the esophageal cancer group were significantly higher than those in the healthy group $(\mathrm{P}<0.05$; Table 1$)$.

\section{Comparison of CA19-9, Bcl-2, and CYFRA21-1 levels in esophageal cancer patients with different clinical features}

The levels of CA19-9, Bcl-2, and CYFRA21-1 in patients with poorly differentiated, stage III-IV carcinoma and lymph node metastasis were significantly higher than those in patients with moderately well differentiated, stage I-II carcinoma without lymph node metastasis $(\mathrm{P}>0.05$; Table 2$)$.

\section{MSCT signs of esophageal carcinoma}

The CT scans of patients in the esophageal cancer group showed esophageal walls with irregular, needle-shaped, annular or localized eccentric thickening and narrowed lumens, which were dilated above the cancerous lesions. Some tumors compressed adjacent organs to deform and shift the organs, resulting in the disappearance of surrounding fat layers. Enhanced scans showed mild or moderate enhancement, with large diameter lesions unable to enhance central, low-density, necrotic areas. Among 53 patients with lymph node metastasis, 26 patients had lymph node metastasis of the right recurrent laryngeal nerve, which was consistent with the pathological report. The average short diameter of metastatic lymph nodes was $(0.42 \pm 0.04) \mathrm{cm}$, and the average long diameter was $(0.69 \pm 0.11) \mathrm{cm}$. Typical case images are shown in Figure $1 A-E$.

\section{Diagnostic value of MSCT combined with CA19-9, Bcl-2, and CYFRA21-1 detection in esophageal cancer}

The ROC curve showed that the area under the curve (AUC) and the sensitivity and specificity of MSCT combined with CA19-9, Bcl-2, and CYFRA21-1 detection were higher than for esophageal lesions detected by individual indicators. See Table 3 and Figure 2. 
Table 2 Comparison of CA19-9, Bcl-2, and CYFRA21-1 levels in esophageal cancer patients with different clinical features

\begin{tabular}{|c|c|c|c|c|}
\hline Clinical characteristics & Cases & CA19-9 (U/mL) & $\mathrm{Bcl}-2$ (ng/mL) & CYFRA21-1 $(\mu / \mathrm{mL})$ \\
\hline Non-squamous & 51 & $32.73 \pm 10.04$ & $17.66 \pm 4.99$ & $5.11 \pm 2.92$ \\
\hline Squamous cell carcinoma & 23 & $33.72 \pm 11.11$ & $17.68 \pm 4.71$ & $5.21 \pm 3.06$ \\
\hline \multicolumn{5}{|l|}{ Differentiation extent } \\
\hline Poorly differentiated & 41 & $41.94 \pm 2.62^{*}$ & $21.39 \pm 3.08^{\star}$ & $7.51 \pm 1.68^{*}$ \\
\hline \multicolumn{5}{|l|}{ TNM stage } \\
\hline I-II & 22 & $21.51 \pm 2.76$ & $13.19 \pm 1.70$ & $2.18 \pm 0.29$ \\
\hline III-IV & 52 & $37.91 \pm 8.23^{\#}$ & $19.56 \pm 4.53^{\#}$ & $6.39 \pm 2.65^{\#}$ \\
\hline No & 21 & $21.53 \pm 2.82^{\&}$ & $13.10 \pm 1.68^{\&}$ & $2.20 \pm 0.29^{\&}$ \\
\hline
\end{tabular}

*, compared with medium-high differentiation; ", compared with stage I-II; *, compared with lymph node metastasis, P<0.05. CA19-9, carbohydrate antigen 19-9; Bcl-2, B-cell leukemia/lymphoma-2 protein; CYFRA21-1, cytokeratin 19 fragment antigen 21-1.

\section{Discussion}

In recent years, esophageal cancer has become one of the most significant malignant diseases threatening human life and health. However, at present, there is a lack of means for making an early diagnosis of esophageal cancer in the clinic, and the symptoms of early disease are non-specific. Hence, most patients are diagnosed in the middle and late stages of disease when the effect of treatment is poor $(6,7)$. Therefore, to improve the outcomes of esophageal cancer, it is necessary to not only improve the patient's awareness of the importance of timely treatment but to also identify the appropriate investigations to support an early diagnosis. This will enable patients to receive effective early intervention to delay the progression of disease, improving the prognosis of patients with esophageal cancer $(8,9)$.

CA19-9 is an oligosaccharide, tumor-related antigen. The expression of CA19-9 in normal serum is low, while its expression in various malignant tumors, such as tumors of the pancreas and the stomach (10), is high. However, CA199 is also expressed in other medical conditions, such as acute and chronic pancreatitis, cholecystitis, and cirrhosis (11). Therefore, it cannot be used as a specific indicator for the diagnosis of esophageal cancer. Bcl-2 is an important antiapoptotic factor, functioning in the mitochondrial pathway to inhibit cell apoptosis. The process of tumor cell apoptosis is abnormal and provides an opportunity for the malignant transformation of cells (12). Nougarede et al. (13) found that Bcl-2 was highly expressed in esophageal cancer, which plays an important role in tumorigenesis and development.

CYFRA21-1 is a cytokeratin 19 fragment mainly distributed in the tumor cell-associated cytoplasm of lung cancer, esophageal cancer, and other cancers of epithelial origin. Takeuchi et al. (14) found that the serum level of CYFRA21-1 was significantly increased when cells were cancerous. Kammer et al. (15) also found that the positive rate of CYFRA21-1 in tumors was higher than that of squamous cell carcinoma antigen, C-reactive protein and other known tumor markers. In this study, the serum levels of CA19-9, Bcl-2, and CYFRA21-1 in patients with esophageal cancer were higher than those in the healthy group. Furthermore, tumor biomarker levels differed between patients according to the degree of cancer cell differentiation, clinical stage of disease, and extent of lymph node metastasis. Hence, these tumor markers can be used in a preliminary assessment of esophageal cancer and related conditions. Although tumor markers have the potential to contribute to the diagnosis of malignant diseases and the evaluation of disease condition and prognosis, they are susceptible to various factors and can contribute to false-positive or false-negative readings, providing false information for clinical diagnosis. Although combined with the detection of multiple markers, it is still unable to obtain accurate diagnostic results $(16,17)$. 

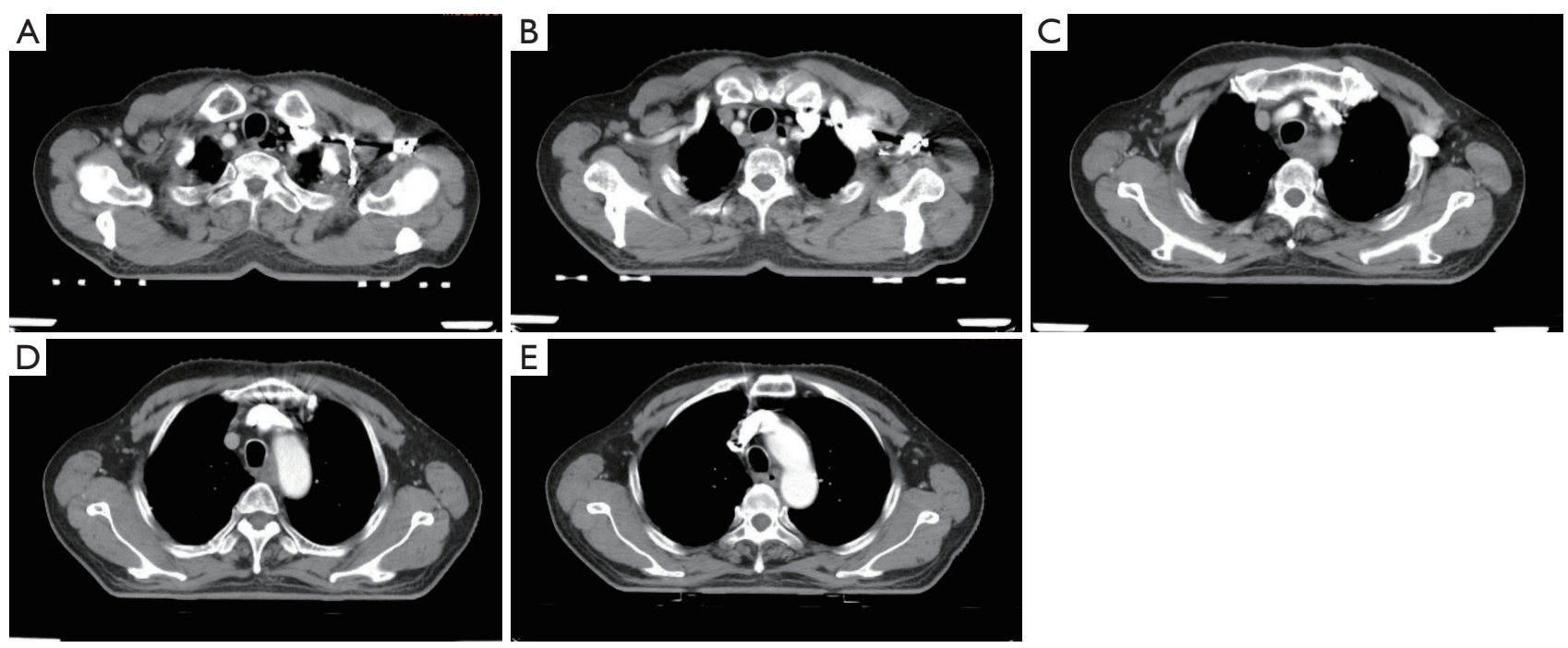

Figure 1 A 68-year-old male presented with middle and upper esophageal cancer. The chest-enhanced computed tomography (CT) imaging showed thickening of the middle and upper esophageal wall, of approximately $1.7 \mathrm{~cm}$, and stenosis of the lumen (A,B). After enhancement, there was mild to moderate inhomogeneous continuous enhancement (C-E).

Table 3 Diagnostic value of MSCT combined with CA19-9, Bcl-2, and CYFRA21-1 in esophageal cancer

\begin{tabular}{|c|c|c|c|c|c|}
\hline Predictor & AUC & Standard error & Progressive Sig & \multicolumn{2}{|c|}{ Progressive $95 \% \mathrm{Cl}$} \\
\hline CA19-9 & 0.851 & 0.033 & $<0.001$ & 0.786 & 0.915 \\
\hline $\mathrm{Bcl}-2$ & 0.738 & 0.045 & $<0.001$ & 0.649 & 0.826 \\
\hline CYFRA21-1 & 0.747 & 0.043 & $<0.001$ & 0.663 & 0.830 \\
\hline Combined & 0.959 & 0.016 & $<0.001$ & 0.927 & 0.990 \\
\hline
\end{tabular}

MSCT, multi-slice spiral computed tomography; AUC, areas under the curve; CA19-9, carbohydrate antigen 19-9; Bcl-2, B-cell leukemia/ lymphoma-2 protein; CYFRA21-1, cytokeratin 19 fragment antigen 21-1.

Relevant reports have found that the accuracy of disease diagnosis can be improved by comprehensive imaging, laboratory examination, and other means (18). MSCT is a commonly used imaging method for the early clinical diagnosis of esophageal cancer. Compared with MRI and X-ray, MSCT has many advantages, such as being simple to operate while enabling high-density resolution and fast scanning. The location, size and relationship with surrounding tissues can be clearly displayed in an esophageal cancer examination. International studies have confirmed that MSCT can support a preliminary assessment of esophageal cancer (19). However, a CT examination also has certain limitations. For example, the enhanced scanning time is difficult to grasp, which impacts the display of the lesion, and is limited by the scanning range; hence, CT imaging has little diagnostic significance in the staging of esophageal cancer (20).

In this study, MSCT and the detection of CA19-9, Bcl2, and CYFRA21-1 were combined to diagnose esophageal cancer cases. The ROC results showed that the AUC and the sensitivity and specificity of the combined approach to diagnosis were higher than those of a single approach to diagnosis, suggesting that the combined approach to diagnosis can effectively improve the diagnosis of esophageal cancer. It is speculated that that the combination of MSCT and serum factor detection provides a dual basis for the clinical diagnosis of esophageal cancer, which effectively compensates for the lack of a single diagnosis 


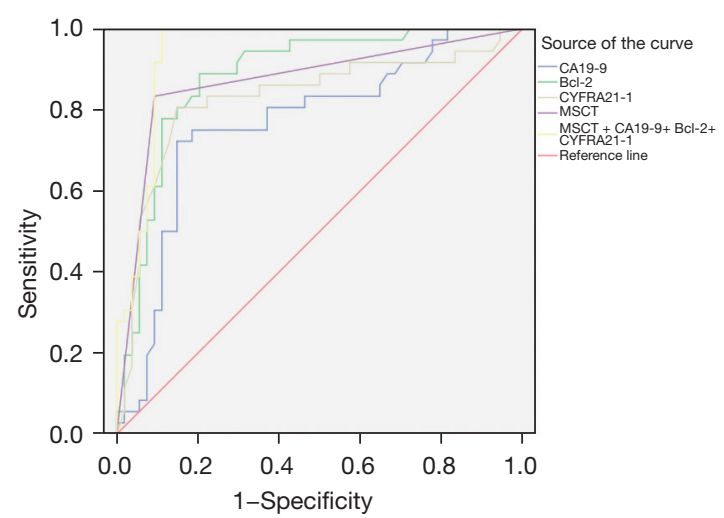

Figure 2 ROC curve analysis of MSCT combined with CA199, Bcl-2 and CYFRA21-1 detection in the diagnosis of esophageal cancer. ROC, receiver operating characteristic; MSCT, multislice spiral computed tomography; CA19-9, carbohydrate antigen 19-9; Bcl-2, B-cell leukemia/lymphoma-2 protein; CYFRA21-1, cytokeratin 19 fragment antigen 21-1.

and improves the diagnostic accuracy, which is of great significance in clinical diagnosis and treatment.

In summary, serum levels of CA19-9, Bcl-2, and CYFRA21-1 were abnormally expressed in patients with esophageal cancer, which may be related to the occurrence and development of esophageal cancer. The above factors combined with the MSCT examination can improve the diagnostic value of esophageal cancer.

\section{Acknowledgments}

Funding: None.

\section{Footnote}

Reporting Checklist: The authors have completed the STARD reporting checklist. Available at https://dx.doi. org/10.21037/tcr-21-2522

Data Sharing Statement: Available at https://dx.doi. org/10.21037/tcr-21-2522

Conflicts of Interest: All authors have completed the ICMJE uniform disclosure form (available at https://dx.doi. org/10.21037/tcr-21-2522). The authors have no conflicts of interest to declare.

Etbical Statement: The authors are accountable for all aspects of the work in ensuring that questions related to the accuracy or integrity of any part of the work are appropriately investigated and resolved. All procedures performed in this study involving human participants were in accordance with the Declaration of Helsinki (as revised in 2013). The study was approved by ethics committee of Dazu District People's Hospital (No. EC2020-031-05) and informed consent was taken from all the patients.

Open Access Statement: This is an Open Access article distributed in accordance with the Creative Commons Attribution-NonCommercial-NoDerivs 4.0 International License (CC BY-NC-ND 4.0), which permits the noncommercial replication and distribution of the article with the strict proviso that no changes or edits are made and the original work is properly cited (including links to both the formal publication through the relevant DOI and the license). See: https://creativecommons.org/licenses/by-nc-nd/4.0/.

\section{References}

1. Su HA, Hsiao SW, Hsu YC, et al. Superiority of NBI endoscopy to PET/CT scan in detecting esophageal cancer among head and neck cancer patients: a retrospective cohort analysis. BMC Cancer 2020;20:69.

2. Demarest CT, Chang AC. The Landmark Series: Multimodal Therapy for Esophageal Cancer. Ann Surg Oncol 2021;28:3375-82.

3. Kerkhofs M, La Rovere R, Welkenhuysen K, et al. BIRD2, a BH4-domain-targeting peptide of $\mathrm{Bcl}-2$, provokes Bax/Bak-independent cell death in B-cell cancers through mitochondrial Ca2+-dependent mPTP opening. Cell Calcium 2021;94:102333.

4. Goense L, Heethuis SE, van Rossum PSN, et al. Correlation between functional imaging markers derived from diffusion-weighted MRI and 18F-FDG PET/CT in esophageal cancer. Nucl Med Commun 2018;39:60-7.

5. Zheng Q, Zhang L, Tu M, et al. Development of a panel of autoantibody against NSG1 with CEA, CYFRA21-1, and SCC-Ag for the diagnosis of esophageal squamous cell carcinoma. Clin Chim Acta 2021;520:126-32.

6. Wang J, Yang Y, Shaik MS. Prognostic significance of the number of lymph nodes dissection in esophageal adenocarcinoma patients. Transl Cancer Res 2020;9:3406-15.

7. Zopfs D, Große Hokamp N, Reimer R, et al. Value of spectral detector CT for pretherapeutic, locoregional assessment of esophageal cancer. Eur J Radiol 
2021;134:109423.

8. Bakhos CT, Acevedo E Jr, Petrov RV, et al. Surveillance Following Treatment of Esophageal Cancer. Surg Clin North Am 2021;101:499-509.

9. Valkema MJ, van der Wilk BJ, Eyck BM, et al. Surveillance of Clinically Complete Responders Using Serial 18F-FDG PET/CT Scans in Patients with Esophageal Cancer After Neoadjuvant Chemoradiotherapy. J Nucl Med 2021;62:486-92.

10. Kosuke S, Tomotaka S, Kohei N, et al. Utility of PETCT CMR after neoadjuvant chemotherapy with DCF for esophageal cancer as a predictive factor of recurrence. $\mathrm{J}$ Clin Oncol 2020;38:434.

11. Fahrmann JF, Schmidt CM, Mao X, et al. Lead-Time Trajectory of CA19-9 as an Anchor Marker for Pancreatic Cancer Early Detection. Gastroenterology 2021;160:13731383.e6.

12. Akolkar D, Patil D, Page R, et al. Circulating ensembles of tumor-associated cells in gastrointestinal cancers. J Clin Oncol 2020;38:808.

13. Nougarede A, Popgeorgiev N, Kassem L, et al. Breast Cancer Targeting through Inhibition of the Endoplasmic Reticulum-Based Apoptosis Regulator Nrh/BCL2L10. Cancer Res 2018;78:1404-17.

14. Takeuchi A, Oguri T, Sone K, et al. Predictive and Prognostic Value of CYFRA 21-1 for Advanced Non-small Cell Lung Cancer Treated with EGFR-TKIs. Anticancer Res 2017;37:5771-6.

Cite this article as: Hao J, Liu W, Zhao C, Xia T. The diagnostic significance of 64-slice spiral CT combined with serological CA19-9, Bcl-2, CYFRA21-1 detection in thoracic esophageal carcinoma. Transl Cancer Res 2021;10(12):5383-5389. doi: $10.21037 /$ tcr-21-2522
15. Kammer MN, Kussrow AK, Webster RL, et al. Compensated Interferometry Measures of CYFRA 21-1 Improve Diagnosis of Lung Cancer. ACS Comb Sci 2019;21:465-72.

16. Azizian A, Rühlmann F, Krause T, et al. CA19-9 for detecting recurrence of pancreatic cancer. Sci Rep 2020;10:1332.

17. Arcidiacono D, Dedja A, Giacometti C, et al. Hyperinsulinemia Promotes Esophageal Cancer Development in a Surgically-Induced DuodenoEsophageal Reflux Murine Model. Int J Mol Sci 2018;19:1198.

18. Yajima S, Suzuki T, Oshima Y, et al. New Assay System Elecsys Anti-p53 to Detect Serum Anti-p53 Antibodies in Esophageal Cancer Patients and Colorectal Cancer Patients: Multi-institutional Study. Ann Surg Oncol 2021;28:4007-15.

19. Yola ML, Atar N, Özcan N. A novel electrochemical lung cancer biomarker cytokeratin 19 fragment antigen 21-1 immunosensor based on Si3N4/MoS2 incorporated MWCNTs and core-shell type magnetic nanoparticles. Nanoscale 2021;13:4660-9.

20. Mishra A, Singh N, Sahu DK, et al. 33P Expression of biomarkers IDH1, CEA, TPA and CYFRA21-1 in peripheral blood and tissue of non-small cell lung carcinoma patients detected by real-time PCR. J Thorac Oncol 2018;13:S18. 